\title{
Anesthetic considerations for a patient with myasthenia gravis undergoing deep sedation in an outpatient oral surgery setting
}

\author{
Shamit S. Prabhu' ${ }^{1,2}$, Saad A. Khan', Alexander L. Doudnikoff', Uday N. Reebye \\ ${ }^{1}$ Triangle Implant Center, Durham, NC, USA \\ ${ }^{2}$ Wake Forest School of Medicine, Winston-Salem, NC, USA
}

\begin{abstract}
Myasthenia gravis (MG) is a neuromuscular autoimmune disorder which clinically presents as muscular weakness and fatigue due to autoantibody formation against acetylcholine receptors (AChR), leading to their subsequent destruction. Due to the neuromuscular implications of MG, certain considerations must be taken into account when providing anesthesia to MG patients. In the following case report, we have outlined procedural considerations for the anesthetic management of a patient with MG undergoing deep sedation for an elective oral surgery in an outpatient setting, as well as a discussion of relevant literature.
\end{abstract}

Keywords: Anesthesia; Myasthenia Gravis; Oral Surgery.

This is an Open Access article distributed under the terms of the Creative Commons Attribution Non-Commercial License (http://creativecommons.org/licenses/by-nc/4.0/) which permits unrestricted non-commercial use, distribution, and reproduction in any medium, provided the original work is properly cited.

\section{INTRODUCTION}

Myasthenia gravis (MG) is an autoimmune disease of neuromuscular origin whose typical clinical manifestations include symptoms such as skeletal muscle weakness and increased fatigue upon exertion [1]. Early symptomatic signs indicative of MG are typically localized to the ocular, ophthalmic, and facial muscle groups; patients can present with diplopia, photophobia, ptosis, weakness of the orbicularis oculi, dysphagia, poor mastication as a result of weak masseter muscles, and possibly a hoarse speaking voice due to vocal cord paralysis [2]. Progression of MG leads to the involvement of additional muscle groups such as the upper and lower extremities, leading to deficits in the ability to complete basic functional motor tasks such as raising their arms over their head, walking up or down stairs, and getting in and out of a chair, to name a few [2]. If left untreated, the disease will manifest as respiratory muscle weakness preceding respiratory failure, triggering what is known as a myasthenic crisis [3]. MG patients are considered to be in myasthenic crisis if they are unable to maintain patency of their airway due to respiratory insufficiencies, thus requiring artificial ventilation [2-4].

The symptoms involved in the pathogenesis of MG are a consequence of the immune system forming autoantibodies against postsynaptic membrane nicotinic acetylcholine receptors (AChR) in the neuromuscular junction (NMJ), reducing the ability for skeletal muscles to depolarize and generate muscular contractions [5]. There are a number of mechanistic pathways implicated

Received: November 28, 2018 • Revised: February 7, 2019 • Accepted: February 8, 2019

Corresponding Author: Saad A. Khan, Triangle Implant Center, Durham, 761 Meadowood Drive Burlington, NC 27215, USA

Tel: +1 (336) 693-0586 E-mail: saad.khan92294@gmail.com

Copyright(c) 2019 Journal of Dental Anesthesia and Pain Medicine 


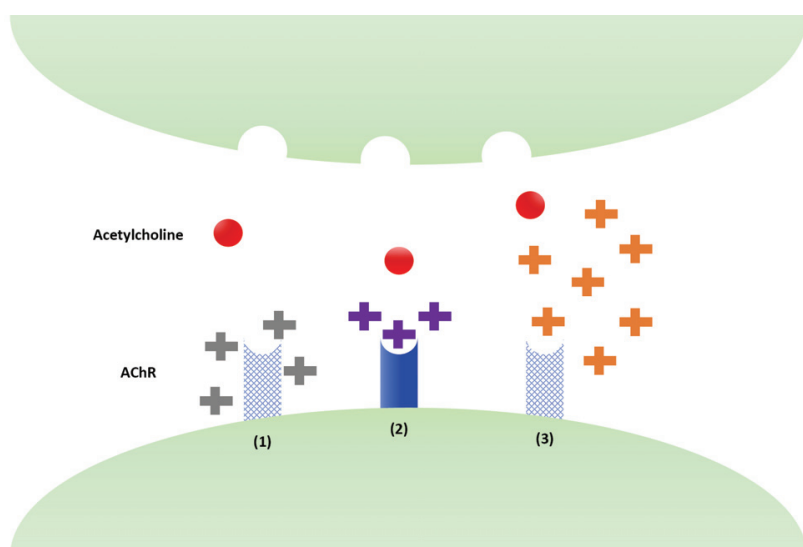

Fig. 1. Pathophysiologic pathways of MG: (1) AChR autoantibody destruction, (2) ACh binding site blockage, (3) Complement-mediated destruction of $\mathrm{AChR}$ and motor end plate.

in the AChR autoimmune response characteristic of MG and other NMJ disorders: the destruction of AChRs, blockage of acetylcholine binding sites, as well as damage caused by the complement cascade all exert inhibitory effects at the molecular level with regards to neurotransmission (Fig. 1) [6,7].

Clinical diagnosis of $\mathrm{MG}$ is contingent upon the integration of the signs and symptoms characteristic of MG with serum laboratory exams testing for the presence of autoantibody profiles implicated in the disease pathology [5]. Given that nearly 85 to 90 percent of MG patients display serum AChR autoantibodies, administration of acetylcholinesterase inhibitors such as edrophonium or neostigmine, colloquially known as the Tensilon test, is the standard diagnostic tool [6]. The pharmacologic action of edrophonium and neostigmine is to prevent the breakdown of acetylcholine, therefore a positive diagnostic confirmation for MG, via the Tensilon test, is indicated by the affected muscle groups displaying renewed strength in muscular contraction post-administration of acetylcholinesterase inhibitors [8].

Management of MG can prove to be challenging given the pathophysiological manifestations of this disease. In particular, such concerns arise when surgical intervention under general anesthesia is necessary to treat an overlying medical issue in patients with MG [9]. The anticipation and subsequent prevention of any potential intraoperative complications such as myasthenic crisis and/or respiratory failure in this patient population are of paramount importance; therefore, special anesthetic considerations must be taken into account during the peri and post-operative periods for $\mathrm{MG}$ patients undergoing surgical procedures. In the following report, we discuss the anesthetic management of a patient with $\mathrm{MG}$ undergoing an outpatient elective oral surgery procedure while under deep sedation.

\section{CASE}

A 49-year-old female patient presented to the oral surgery clinic for extraction of teeth numbers 1 and 16 . Her prior medical history included a myasthenia gravis diagnosis in 2001, as well as hypertension. Her reported therapeutic management for $\mathrm{MG}$ consisted of an immunosuppressant, mycophenolate mofetil $(500 \mathrm{mg}$, twice daily). Her hypertension was treated with the alpha-agonist clonidine $(2 \mathrm{mg})$, thiazide-like diuretic chlorthalidone $(25 \mathrm{mg})$, and potassium-sparing diuretic spironolactone (25 $\mathrm{mg}$, once daily).

She did not display any characteristics indicative of muscular weakness such as ptosis or diplopia during her pre-operative consultation, nor did she describe any dysphagia or dyspnea indicative of airway patency issues. Clinical examination revealed no extraoral or intraoral swelling, edema, or tenderness. Both tooth 1 and tooth 16 were severely carious, positive percussion, and non-restorable. The patient elected to undergo the procedure under deep sedation. Our anesthetic plan included induction with propofol as it provides rapid onset, short duration of action, rapid clearance, and moderate suppression of airway reflexes. It was noted to avoid any use of neuromuscular blocking agents (NMBAs) unless they were absolutely required due to the fact MG patients are highly sensitive to the effect of muscle relaxants. If deemed necessary for the procedure, a small dose of a non-depolarizing NMBA, such as rocuronium, titrated to effect followed by reversal with sugammadex would be utilized. 


\section{Anesthetic Procedure}

Prior to the operation, the patient had a nasal cannula, blood pressure cuff, 6 lead ECG, and pulse oximeter placed to deliver oxygen and measure carbon dioxide emissions, blood pressure, heart rate, and oxygen saturation, pre, peri, and post-operatively. Intravenous access was obtained in the left antecubital fossa by our anesthesiologist. The patient was induced with $137 \mathrm{mg}$ of propofol titrated to effect and $50 \mu \mathrm{g}$ fentanyl. Her respiratory rate slowed when administered the initial bolus but returned to normal levels. After the patient was induced, surgical sites \#1 and \#16 were each infiltrated with 2 carpules of $2 \%$ lidocaine buffered with $1: 100,000$ epinephrine to achieve bilateral posterior superior alveolar nerve block as well as palatal and local infiltration nerve blocks. One carpule of $0.5 \%$ bupivacaine hydrochloride with 1:200,000 epinephrine was divided between both surgical sites to provide extended local anesthesia. Sedation was maintained through the IV, and her airway was manually supported with a jaw thrust maneuver utilized as needed.

\section{Surgical Procedure}

A \#15 blade was used to make a sulcular incision along the mid-alveolar, crestal bone, right and left maxillary tuberosity, and the area of teeth number 1 and 16. A full thickness mucoperiosteal flap was elevated onto the buccal aspect. A straight elevator was positioned between the alveolus and the root surface. The teeth were elevated and the periodontal ligament was separated from the alveolus. Both teeth were easily extracted using a No. 150 upper universal forcep. A bone file was used to smooth the bone alveolus, and then curetted and irrigated with normal saline solution. A $4 \times 4$ sterile gauze was applied at both surgical sites to aid in hemostasis. The sedation duration lasted for 13 minutes, and the patient awoke independently from anesthesia. She had no apneic events throughout the operation. The patient was drowsy, but alert, and was taken to the recovery room where she remained until her vital signs stabilized.

\section{DISCUSSION}

Osserman was the first to classify MG into different degrees of severity and localization, and this classification system has since served as a useful and practical methodology in the clinic when managing patients with MG [10]. This system addresses the variability associated with MG not only in terms of the extent of muscle weakness but also delineates between combinations of affected muscle groups $[2,10]$. As follows, MG can be classified into five different subgroups that increase in degree of severity: localized ocular symptoms (Group I), mild to moderate generalized muscle weakness (Group IIA and IIB), severe generalized MG with characteristics such as fulminant hepatic failure with or without respiratory complications (Group III), and complete muscular atrophy with resulting myasthenic crisis due to respiratory failure (Group IV) [2,10].

There are a number of therapeutic modalities that can be utilized in conjunction with MG patient management. Cholinesterase inhibitors are first-line medications in the treatment of MG, the anticholinesterase drug pyridostigmine (Mestinon) being one of the more commonly administered drugs in this regard, with a dosing rate ranging anywhere from 30 to $60 \mathrm{mg}$ every 4 to 6 hours, or a maximum dose of $120 \mathrm{mg}$ per 3 hours $[11,12]$. Immunosuppressants aid in the circumvention of the body's immune response targeting AChRs, thereby improving neuromuscular transmission as a result [9]. Corticosteroids have also been shown to be effective, functioning in a similar manner as immunosuppressants in dampening the immune response at the motor end plate $[9,13]$. From an operative standpoint, there are varying opinions as far as medication usage prior to a procedure with specific regards to anticholinesterases. The amount of anticholinesterase administered pre-operatively is a function of the necessity and dosage required of muscle relaxants for the particular procedure, with dosages possibly being withheld or reduced [14]. Corticosteroids can either be weaned off in MG patients, or those with 
long-term use of steroid treatment can receive one intravenous dose prior to surgery, as acute cessation of usage can contribute to perioperative hemodynamic instability [15].

When considering the anesthetic implications of performing surgical procedures on MG patients, proper preoperative evaluation is critical in ensuring that operative complications do not arise. In that regard, assessing the patency of the patient's airway and overall respiratory muscle strength is critical. The clinician should be aware and observe for possible signs of bulbar symptoms or respiratory muscle involvement prior to operating on patients with $\mathrm{MG}$, both of which pose risks for the post-operative respiratory state of the patient [9]. The patient should also exhibit normal cardiovascular function and present without any cardiac arrhythmias such as bradycardia or atrial fibrillation, ST and T wave abnormalities, and conductive deficits [14]. Coupling these assessments with a survey of the patient's degree of severity of MG, muscle groups involved, and their longitudinal disease management, as well as taking into account their current medications, ensures a correct and safe operative management plan is in place tailored both to the patient and the procedure being performed [15]. Post-operatively, it is important the patient exhibits unassisted ventilation with a patent airway, should be monitored for any signs of respiratory distress, and display no symptoms indicative of myasthenic or cholinergic crisis $[13,15]$.

Management of the patient's airway was achieved by utilizing a head tilt/chin lift for spontaneous intervention with usage of a jaw thrust to provide additional pain stimulus, as needed. Provided that the preoperative evaluation of the patient yields no risk factors or contraindications for deep sedation, this is a preferable method for airway management as compared to paralysis, which greatly increases the risk for perioperative myasthenic crisis. In the context of dental procedures, invasive airway devices donot necessarily have to be avoided if paralysis is required for placement as they can always be repositioned with minimal risk. A nasal intubation can be accomplished if the dental practitioner wants to avoid potential visual blockage of the surgical field.

Sedation is common practice in dentistry and is utilized for many routine procedures, and a key distinction is the difference between conscious and deep sedation. The former describes a situation where the patient receiving treatment is under sedation but still alert and cooperative, while the latter describes a loss of consciousness in the patient [16]. Respiratory depression is a common side effect associated with deep sedation, and can be exacerbated in patients with MG [14]. As such, close observation of MG patients for any signs of perioperative respiratory distress that could trigger a myasthenic crisis is of paramount importance given their predisposed neuromuscular issues $[14,15]$.

A number of drugs and anesthetics regularly used in dentistry and oral surgery are contraindicated with MG patients due to the increased risk they present in exacerbating the MG-associated muscular symptoms [1]. Drugs that interfere with neuromuscular transmission or cause respiratory depression, thereby increasing the risk of myasthenic crisis, are to be avoided [9,11]. Namely, non-depolarizing NMBAs have an inhibitory effect on neuromuscular transmission in MG patients and as such should be utilized with caution for procedures $[9,13]$. Low doses titrated to effect of short or intermediate-acting non-depolarizing NMBAs, such as rocuronium, are preferable to their longer-acting counterparts to help minimize the suppressive effects on neurotransmission, and is easily reversible with sugammadex $[9,15]$. Moreover, depolarizing NMBAs such as succinylcholine are met with resistance when utilized with MG patients due to the diminished presence of available AChRs, which in turn can cause adverse and prolonged neuromuscular blockade given the majority of MG patients utilize anticholinesterases as their first line treatment [15]. As such, succinylcholine is not recommended for use in an operative setting.

With regards to local anesthetics, ester-type local anesthetics such as procaine are contraindicated in $\mathrm{MG}$ 
patients due to their hydrolytic susceptibility by cholinesterases, while amide-type variety anesthetics such as mepivacaine, bupivacaine, or lidocaine are safe to utilize [11]. Local anesthetics of the amide-type variety such as mepivacaine or xylocaine, which was used on our patient, are safe to utilize [11]. In lieu of bilateral mandibular blocks, we utilized a combination of local infiltration injection techniques in concert with a bilateral posterior superior alveolar nerve block, thereby alleviating the potential risk for swallowing-related issues during the procedure [12].

Sedation with nitrous oxide-oxygen is safe to utilize in MG patients, and also provides anxiolytic benefits to counteract the possibility for surgery-associated emotional distress that can potentially trigger a myasthenic crisis [9,11]. Additionally, inhalational anesthetics such as desflurane, isoflurane, sevoflurane, and halothane are sufficient for induction [13]. Inductive agents such as propofol and fentanyl are safe substitutes that circumvent the possibility of myasthenic exacerbation, and are preferred for their short duration, minimal neuromuscular effects, and can be easily reversed in the event a crisis is precipitated. $[9,13,14]$.

While a literature review revealed some strategies for the management of MG, few discuss considerations in the context of outpatient dental settings. Furthermore, to the best of our knowledge, no published case reports describing the management of MG patients in an outpatient dental setting were found. Our successful management strategy of utilizing a limited number of sedation agents with rapid metabolism, combined with maintenance of spontaneous respirations, emphasized the importance of considering simplicity and pre-operative preparation as a goal when safely managing MG patients.

\section{AUHHOR ORGIDS}

Shamit S. Prabhu: https://orcid.org/0000-0001-5954-4748

Saad A. Khan: https://orcid.org/0000-0002-7082-5230

\section{REFERENCES}

1. Tamburrini A, Tacconi F, Barlattani A, C Mineo T. An update on myasthenia gravis, challenging disease for the dental profession. J Oral Sci 2015; 57: 161-8.

2. Keesey JC. Clinical evaluation and management of myasthenia gravis. Muscle Nerve 2004; 29: 484-505.

3. Thavasothy M, Hirsch N. Myasthenia gravis. BJA CEPD Reviews 2002; 2:88-90. Available from: http://dx.doi.org/ $10.1093 /$ bjacepd/2.3.88

4. Wendell LC, Levine JM. Myasthenic crisis. Neurohospitalist 2011; 1: 16-22.

5. Gilhus NE. Myasthenia Gravis. N Engl J Med 2016; 375: 2570-81.

6. Drachman DB. Myasthenia Gravis. N Engl J Med 1994; 330: $1797-810$.

7. Ha JC, Richman DP. Myasthenia gravis and related disorders: Pathology and molecular pathogenesis. Biochim Biophys Acta 2015; 1852: 651-7.

8. Daroff RB. The office Tensilon test for ocular myasthenia gravis. Arch Neurol 1986; 43: 843-4.

9. Jamal BT, Herb K. Perioperative management of patients with myasthenia gravis: Prevention, recognition, and treatment. Oral Surg Oral Med Oral Pathol Oral Radiol Endod 2009; 107: 612-5.

10. Osserman K. Myasthenia Gravis. Grune \& Stratton. 1958. p. $78-80$.

11. Yarom N, Barnea E, Nissan J, Gorsky M. Dental management of patients with myasthenia gravis: A literature review. Oral Surgery Oral Med Oral Pathol Oral Radiol Endodontology 2005; 100: 158-63.

12. Patil PM, Singh G, Patil SP. Dentistry and the myasthenia gravis patient: A review of the current state of the art. Oral Surg Oral Med Oral Pathol Oral Radiol 2012; 114: e1-8.

13. Abel M, Eisenkraft JB. Anesthetic implications of myasthenia gravis. Mt Sinai J Med 2002; 69: 31-7.

14. Ceremuga TE, Yao XI, McCabe JT. Etiology, mechanisms, and anesthesia implications of autoimmune myasthenia gravis. AANA J 2002; 70: 301-10. 
15. Muckler VC, O’Brien JM, Matson SE, Rice AN. Perianesthetic implications and considerations for myasthenia gravis. J Perianesth Nurs 2019; 34: 4-15.
16. Fukayama H. Which Is Better-Conscious Sedation or Deep Sedation? Anesth Prog 1995; 42: 100-2. 